\title{
Staphylococcus aureus and Escherichia coli have disparate dependences on KsgA for growth and ribosome biogenesis
}

\author{
Heather C O'Farrell ${ }^{1}$ and Jason P Rife ${ }^{1,2^{*}}$
}

\begin{abstract}
Background: The KsgA methyltransferase has been conserved throughout evolution, methylating two adenosines in the small subunit rRNA in all three domains of life as well as in eukaryotic organelles that contain ribosomes. Understanding of KsgA's important role in ribosome biogenesis has been recently expanded in Escherichia coli; these studies help explain why KsgA is so highly conserved and also suggest KsgA's potential as an antimicrobial drug target.
\end{abstract}

Results: We have analyzed KsgA's contribution to ribosome biogenesis and cell growth in Staphylococcus aureus. We found that deletion of ksgA in S. aureus led to a cold-sensitive growth phenotype, although KsgA was not as critical for ribosome biogenesis as it was shown to be in E. coli. Additionally, the ksgA knockout strain showed an increased sensitivity to aminoglycoside antibiotics. Overexpression of a catalytically inactive KsgA mutant was deleterious in the knockout strain but not the wild-type strain; this negative phenotype disappeared at low temperature.

Conclusions: This work extends the study of KsgA, allowing comparison of this aspect of ribosome biogenesis between a Gram-negative and a Gram-positive organism. Our results in S. aureus are in contrast to results previously described in E. coli, where the catalytically inactive protein showed a negative phenotype in the presence or absence of endogenous KsgA.

Keywords: KsgA, Ribosome biogenesis, Staphylococcus aureus, Escherichia coli, Methyltransferase

\section{Background}

Ribosome biogenesis in bacteria involves a small number of extra-ribosomal biogenesis factors [1]. Depletion or loss of many of these factors leads to impaired ribosome assembly, and in many cases leads to growth defects or even loss of virulence in pathogenic bacteria. Understanding ribosome biogenesis in bacteria is an active field of study; the bulk of this work has taken place in the model organism Escherichia coli, a Gram-negative $\gamma$-proteobacterium, while lesser study has occurred in other organisms, principally the Gram-positive organism Bacillus subtilis. One ribosome biogenesis factor in particular, KsgA, has been studied intensively for many years in $E$. coli. KsgA dimethylates each

\footnotetext{
* Correspondence: jason.rife@vcu.edu

'Department of Physiology and Molecular Biophysics, Virginia Commonwealth University, Richmond, VA, USA

${ }^{2}$ Institute for Structural Biology and Drug Discovery, Virginia Commonwealth

2Institute for Structural Biology
University, Richmond, VA, USA
}

\section{Biomed Central}

of two adenosines in the 3'-proximal helix (helix 44) of the small subunit rRNA [2] and serves as an important checkpoint in the assembly of the 30S subunit [3]. Cells lacking functional KsgA are often disadvantaged for growth when compared to wild-type cells. Specifically, knockout or mutation of ksgA in the organisms E. coli [3], B. subtilis [4], Mycobacterium tuberculosis [5], Yersinia pseudotuberculosis [6], Chlamydia trachomatis [7] and Erwinia amylovora [8] is deleterious to cell growth, producing strains that either grow slower than or are unable to compete efficiently with wild-type strains. In addition, knockout of $k s g A$ in $Y$. pseudotuberculosis confers an attenuated virulence phenotype on the knockout strain [6]; inactivating mutations of $k s g A$ in the plant pathogen E. amylovora decrease virulence [8].

A key observation to come out of the body of work on KsgA is that overexpression of catalytically inactive KsgA produces a dominant negative phenotype, being deleterious to both ribosome biogenesis and cell growth, thus 
suggesting KsgA might serve as a potential antimicrobial drug target [3]. In this context KsgA and its role in ribosome biogenesis and growth have been studied most extensively in $E$. coli. While $k s g A$ gene knockouts have been tangentially studied in other organisms, no systematic study has been made of KsgA and its role in ribosome biogenesis and growth in another bacterial organism. In order to expand our knowledge of this system, we have extended studies of KsgA into the important Gram-positive human pathogen Staphylococcus aureus.

\section{Results}

\section{Knockout of ksgA leads to a cold-sensitive phenotype}

To investigate the role KsgA plays in ribosome assembly and growth we generated an in-frame deletion of the $k s g A$ gene in the S. aureus strain RN4220. The knockout strain was resistant to the antibiotic kasugamycin (Table 1); this resistant phenotype is also seen in E. coli. We confirmed the loss of KsgA activity in the cell by assaying purified $30 \mathrm{~S}$ ribosomal subunits from both the wild-type (RN) and the knock-out $(\Delta k s g A)$ strains for their ability to be methylated by exogenously added KsgA (Figure 1). As expected, subunits from the RN strain could not be further methylated by recombinant E. coli KsgA, while subunits from the $\Delta k s g A$ strain could be efficiently methylated, albeit not to the same extent as $E$. coli $30 S$ subunits. In addition to confirming the gene deletion, this experiment demonstrated that the structural requirements for KsgA binding to and methylating the small ribosomal subunit are conserved between E. coli and S. aureus.

Next we compared the growth rates of the $\Delta k s g A$ strain and the parental RN cells. We grew both strains in liquid media at a variety of temperatures (Additional file 1) and calculated the doubling times for each strain, shown in Table 2. The strains grew at similar rates at $30^{\circ} \mathrm{C}, 37^{\circ} \mathrm{C}$, and $42^{\circ} \mathrm{C}$. However, at the lower temperatures of $25^{\circ} \mathrm{C}$ and $15^{\circ} \mathrm{C}$ the $\Delta k s g A$ strain grew significantly slower than the RN strain. We can conclude from these data that while knockout of $k s g A$ does not affect cell growth using our test conditions at and around human physiological temperatures the cells become cold-sensitive upon loss of KsgA.

Table 1 Antibiotic resistance of RN4220 and $\Delta k s g A$ strains

\begin{tabular}{lcc}
\hline & \multicolumn{2}{c}{ MIC $(\boldsymbol{\mu g} / \mathbf{m l})$} \\
\cline { 2 - 3 } & $\mathbf{R N 4 2 2 0}$ & $\Delta \mathbf{k s} \boldsymbol{A}$ \\
\hline Kasugamycin & 800 & $>3200$ \\
Kanamycin & 4 & 2 \\
Paromomycin & 4 & 2 \\
Streptomycin & 16 & 16 \\
\hline
\end{tabular}

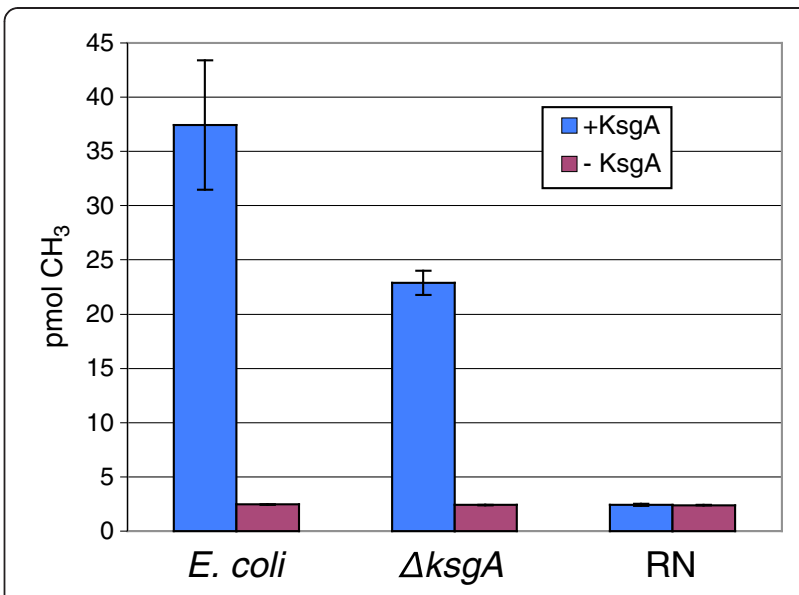

Figure 1 Activity assay. Experiments were performed in triplicate; error bars indicate standard deviation.

We then performed polysome analysis of the ribosomal particles of both strains to ascertain the effects of $k s g A$ knockout on ribosome biogenesis. In these experiments ribosomal material is separated into mature, functional $70 \mathrm{~S}$ ribosomes and free $30 \mathrm{~S}$ and $50 \mathrm{~S}$ subunits. In this way we can visualize increases in immature subunits as a portion of the total ribosomal material. As shown in Figure 2, knockout of $k s g A$ did not result in a significant increase in relative amounts of free $30 \mathrm{~S}$ subunits. Polysome profiles of the $\mathrm{RN}$ and $\Delta k s g A$ strains were similar at $42^{\circ} \mathrm{C}, 37^{\circ} \mathrm{C}$, and $25^{\circ} \mathrm{C}$; the proportion of free subunits increased with lowering temperature in both strains.

Our laboratory previously observed that knockout of $k s g A$ in $E$. coli led to a difference in sensitivity to aminoglycoside antibiotics [9]. Specifically, the $\Delta k s g A$ strain was more sensitive to the 4,6 class of aminoglycosides and less sensitive to 4,5-aminoglycosides, with no change in sensitivity to the aminoglycoside streptomycin. We performed a similar experiment in S. aureus, growing the RN and $\triangle k s g A$ strains on increasing amounts of the antibiotics kanamycin (a 4,6 aminoglycoside), paromomycin (a 4,5-aminoglycoside) and streptomycin (Table 1). The $\Delta k s g A$ strain was more sensitive to both kanamycin and paromomycin, with no change in sensitivity to streptomycin.

Table 2 Doubling times of RN4220 and $\Delta$ ksgA strains

\begin{tabular}{lcc}
\hline & \multicolumn{2}{c}{ Doubling time (min) } \\
\cline { 2 - 3 } & $\mathbf{R N 4 2 2 0}$ & $\boldsymbol{\Delta} \mathbf{k s g}$ \\
\hline $15^{\circ} \mathrm{C}$ & $408.2 \pm 18.2$ & $473.0 \pm 17.2$ \\
$25^{\circ} \mathrm{C}$ & $82.1 \pm 4.1$ & $93.4 \pm 2.0$ \\
$30^{\circ} \mathrm{C}$ & $48.5 \pm 0.6$ & $50.2 \pm 2.2$ \\
$37^{\circ} \mathrm{C}$ & $39.2 \pm 1.8$ & $39.4 \pm 1.7$ \\
$45^{\circ} \mathrm{C}$ & $50.6 \pm 1.5$ & $54.3 \pm 3.5$ \\
\hline
\end{tabular}




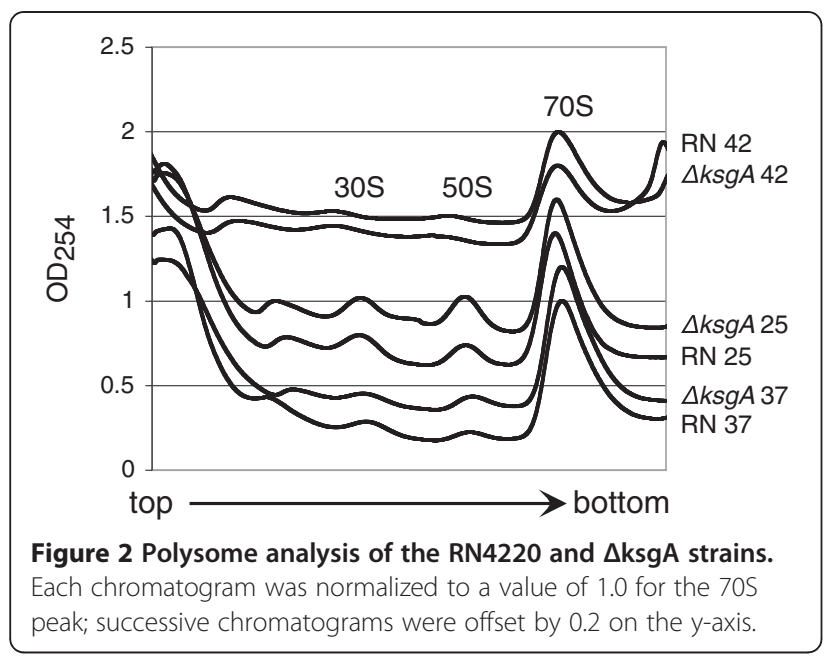

\section{Overexpression of catalytically inactive $\mathrm{KsgA}$ is deleterious}

Overexpression of KsgA, as well as a catalytically inactive mutant of KsgA, was deleterious to $E$. coli growth rates under a variety of conditions [3]. In order to see if these results extended to $S$. aureus we cloned the $k s g A$ gene from the RN4220 strain and constructed the equivalent mutation, E79A. We expressed both WT and E79A protein in $\mathrm{RN}$ and $\triangle k s g A$ cells, using the empty vector (pCN) as a control. Growth experiments were performed as in the previous section (Additional file 2), except that cells were grown in the presence of erythromycin (for plasmid maintenance) and $\mathrm{CdCl}_{2}$ (for protein induction). Under these conditions, the difference in growth rate between the $\mathrm{RN}$ and $\Delta k s g A$ cells expressing the empty vector was not significant, even at $25^{\circ} \mathrm{C}$. Doubling times for each strain are shown in Table 3.

Overexpression of wild-type KsgA did not affect cell growth under any of the conditions we tested. Overexpression of the E79A mutant in cells lacking $k s g A$ had a negative impact on doubling time, but only in the absence of WT enzyme. This effect was seen at $37^{\circ} \mathrm{C}$ but not at $25^{\circ} \mathrm{C}$. In the RN strain, which expresses endogenous KsgA,

Table 3 Doubling times of RN4220 and $\triangle \mathrm{ksgA}$ strains containing PCN constructs

\begin{tabular}{ccc}
\hline & \multicolumn{2}{c}{ Doubling time (min) } \\
\cline { 2 - 3 } & $\mathbf{2 5}^{\circ} \mathbf{C}$ & $\mathbf{3 7}^{\circ} \mathbf{C}$ \\
\hline RN4220 pCN51 & $95.5 \pm 13.8$ & $40.5 \pm 2.7$ \\
pCN-WT & $94.9 \pm 11.0$ & $39.6 \pm 2.4$ \\
pCN-E79A & $92.6 \pm 9.5$ & $39.2 \pm 4.7$ \\
$\Delta$ ksgA pCN51 & $106.1 \pm 11.6$ & $41.4 \pm 2.7$ \\
pCN-WT & $100.0 \pm 8.0$ & $38.3 \pm 2.5$ \\
pCN-E79A & $111.3 \pm 11.5$ & $51.0 \pm 2.3$ \\
\hline
\end{tabular}

overexpression of mutant protein did not significantly affect cell growth.

We next asked if there were any abnormalities in ribosome biogenesis in cells overexpressing WT or mutant KsgA protein. In E. coli overexpression of WT protein led to accumulation of immature $30 \mathrm{~S}$ subunits even when there was no measurable effect on cell growth, and overexpression of the inactive mutant, E66A, resulted in significant effects on ribosome biogenesis in all cases. In S. aureus, overexpression of either WT or E79A protein had very little effect on ribosome biogenesis under any conditions tested (Figure 3), with one exception. The $S$. aureus $\Delta k s g A$ strain overexpressing the E79A mutant protein showed an increase in free subunits relative to the total ribosomal material when grown at $37^{\circ} \mathrm{C}$ but not at $25^{\circ} \mathrm{C}$.

\section{Discussion}

The existence of the $k s g A$ gene was established about forty years ago in E. coli [10]. It was shown to be the sole methyltransferase that converts two adjacent $16 \mathrm{~S}$ rRNA adenosines (A1518 and A1519, E. coli numbering) into $\mathrm{N}^{6}, \mathrm{~N}^{6}$-dimethyladenosines [2], modifications that appeared to hold wide phylogenetic distribution. It is now known that those modifications and the responsible

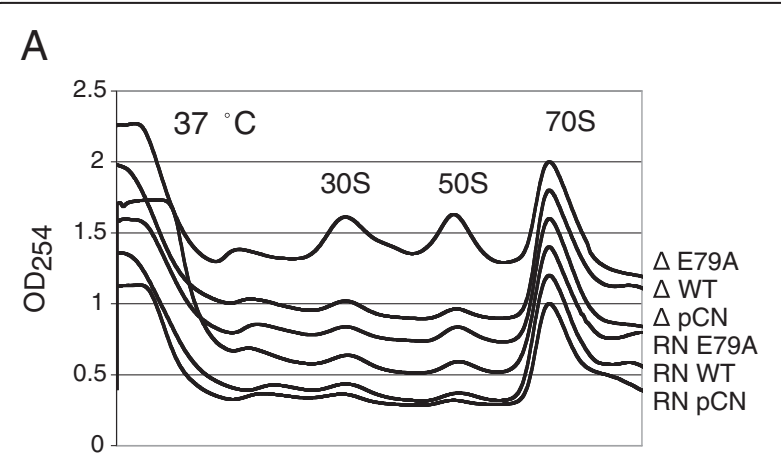

B

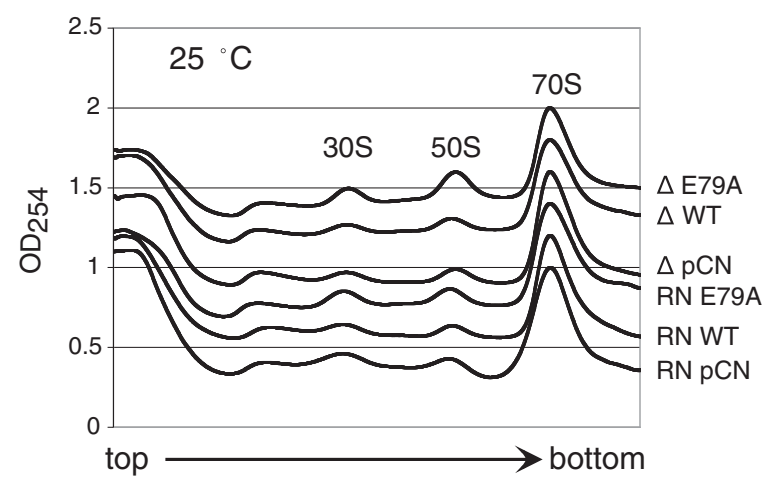

Figure 3 Polysome analysis of the pCN51 strains. Each chromatogram was normalized to a value of 1.0 for the 705 peak; successive chromatograms were offset by 0.2 on the $y$-axis. A) Cells grown at $37^{\circ} \mathrm{C}$. B) Cells grown at $25^{\circ} \mathrm{C}$. 
methyltransferase are all but universally conserved throughout life, thus making KsgA (known as Dim1 in eukaryotes and archaea) a genetic element of the last universal common ancestor. This level of conservation, coupled with the knowledge that KsgA can be dispensed with in several bacteria, albeit with obvious growth defects [3-8], formed the basis of a sharp paradox. If KsgA was not essential, why was it universally conserved? Since evolution is not sentimental, the cellular importance of KsgA and Dim1 was certain but remained to be discovered. In time the stated paradox has partially unraveled. In Saccharomyces cerevisiae (and most likely other eukaryotic organisms) Dim1 is an important member of the rRNA processome, and loss of Dim1 leads to the accumulation of aberrant rRNA species at the expense of functional ribosomes [11]. In E. coli, KsgA serves as a gate-keeper to prevent improperly assembled pre-30S subunits from entering the translation cycle [3]. Under normal conditions, KsgA only provides modest benefit to $30 \mathrm{~S}$ maturation and function. However, KsgA's importance becomes clear under stress conditions, such as growth at cold temperature.

In this work, we sought to define the importance of KsgA to the survivability of the human pathogen S. aureus and to compare our results to those in the model organism E. coli. Somewhat surprisingly, we found that $S$. aureus has a lesser reliance on KsgA under the conditions tested. In E. coli, overexpression of KsgA rescued the cold-sensitive phenotype of $\Delta k s g A$ cells at low temperature but was deleterious for cell growth at $37^{\circ} \mathrm{C}$ in both knockout and parental cells. Overexpression of a catalytically inactive mutant of KsgA, E66A, was deleterious in both strains at both temperatures, even in the presence of endogenous WT protein [3]. We showed that in $S$. aureus the $k s g A$ knock-out strain displayed a slow growth phenotype at low temperature when compared to the parental strain, similar to results in E. coli. However, unlike in E. coli, catalytic inactivation of KsgA's enzymatic function has only mild phenotypic effects, and these effects are not dominant in the presence of WT KsgA. It is noteworthy that the negative growth effect was seen at $37^{\circ} \mathrm{C}$ but not at $25^{\circ} \mathrm{C}$. This result was unexpected, both because $k s g A$ knockout led to cold sensitivity and because negative effects in $E$. coli were exacerbated at low temperature; however, it is possible that growth at the lower temperature results in lower expression of the mutant protein and therefore a smaller negative effect.

In S. aureus, KsgA also appears to be less critical for the assembly of mature ribosomes. Experiments in $E$. coli showed that loss or inactivation of KsgA had obvious effects on ribosome biogenesis even under conditions where a growth phenotype was not apparent [3]. In other words, ribosome biogenesis is sensitive to disruptions in KsgA function that don't affect overall cell growth. We did not see this effect in S. aureus; knockout or inactivation of KsgA resulted in, at most, slight disruption of polysome profiles even under conditions where cell growth was slowed.

On the basis of the data presented here, it would appear that in S. aureus KsgA holds less promise as a drug target than in E. coli. However, we did observe that knockout of $k s g A$ rendered $S$. aureus marginally more sensitive to clinically used aminoglycoside antibiotics, similar to results seen in E. coli. A1518 and A1519 are located distal to the aminoglycoside binding site on the small ribosomal subunit; we therefore hypothesize that effects on antibiotic sensitivity are indirect, likely caused either by conformational or dynamic changes that are propagated from the site of KsgA methylation to the aminoglycoside binding site. This experiment highlights an additional difference between $E$. coli and $S$. aureus ribosomes. While lack of methylation by KsgA leads to increased sensitivity to the 4,6 class of aminoglycosides in both organisms, we see opposite effects on 4,5 aminoglycoside sensitivity. Both the KsgA target site and the aminoglycoside binding site are among the most highly conserved rRNA sequences; it is thus intriguing that distinct effects are seen between the two organisms.

Although ribosome biogenesis has not been well-studied outside of the model organisms $E$. coli and, to a much lesser extent, B. subtilis, it is possible that reported differences in ribosome biogenesis between Gram-negative and Gram-positive organisms are representative of an evolutionary divergence between the two groups of bacteria. One such difference is the case of the ribonuclease RNase III. RNase III is an endonuclease that is involved in processing of the pre-rRNA transcript in both E. coli and $B$. subtilis. However, this enzyme is strictly essential in $B$. subtilis but not in E. coli [12]. Additionally, inactivation of RNase III has different effects on the maturation of $16 \mathrm{~S}$ rRNA in the two organisms [12]. Further work is required to demonstrate whether these results are more broadly applicable in other bacterial species. Our work suggests differences in ribosome biogenesis between $E$. coli and $S$. aureus; it remains to be seen if the differing reliance on KsgA can be defined by a phylogenetic Gram-positive/ Gram-negative split.

KsgA plays a key role in ribosome biogenesis in E. coli, which cannot be separated from its methyltransferase function [3]. Further evidence of KsgA's significance in Gram-negative organisms comes from virulence studies in pathogenic organisms. Disruption of ksgA in Y. pseudotuberculosis confers an attenuated virulence phenotype on the knockout strain [6], and this attenuated strain confers protection against subsequent challenge with the wildtype strain [13]. Additionally, mutation of $\mathrm{ksgA}$ in the plant pathogen E. amylovora decreases virulence [8] and 
disruption of KsgA in S. Enteriditis reduces invasiveness [14]. These studies affirm that KsgA may be a novel drug target in Gram-negative organisms.

Studies on KsgA's role in virulence have not been done in Gram-positive organisms, although in addition to the modest growth defects seen in the $S$. aureus $\Delta k s g A$ strain disruption of the $k s g A$ gene in the Gram-negative Mycobacterium tuberculosis was shown to negatively affect bacterial growth on solid media [5]. It should be noted that disruption of $k s g A$ in $Y$. pseudotuberculosis produced only a slight growth defect and allowed the bacteria to survive in infected mice, even though the strain was not as virulent as the wild-type strain [6]. Likewise, E. amylovora mutants showed reduced virulence despite only small growth defects in vitro and the ability to grow in infected tissue [8]. Further studies will be required to show whether KsgA is similarly correlated with virulence in Gram-positive organisms.

\section{Conclusions}

Given the vital role that the ribosome plays in the cell, it is unsurprising that it is an important target for antibiotic drugs [15]. Although current antibiotic strategies are directed at the functioning of the ribosome, it has been suggested that the ribosome assembly presents a target for novel drug discovery [16]. In support of this hypothesis, knockout of the non-essential ribosome biogenesis factors KsgA and YjeQ, a small-subunit associated GTPase, has been shown to affect bacterial virulence $[6,8,17]$. Therefore, a full understanding of these and other ribosome biogenesis factors in a variety of organisms is critical.

We have extended the study of KsgA into S. aureus and found that KsgA is not as critical for bacterial growth and ribosome biogenesis as was previously shown to be the case in $E$. coli, although the $\Delta k s g A$ knockout does have some negative effects. Additionally, overexpression of the catalytically inactive mutant did not have a dominant effect on growth or ribosome biogenesis in the presence of wild-type protein. Although knockout and mutation of KsgA did not lead to severe growth defects, work in $Y$. pseudotuberculosis and $E$. amylovora suggests that small growth defects in vitro may correlate with larger effects on virulence. Many researchers have suggested that targeting virulence may be a better strategy for antimicrobial therapy than targeting cell growth or viability $[18,19]$. We believe that further research on the role of KsgA in the virulence of $S$. aureus and other pathogens will prove instructive and may provide a viable drug development target.

\section{Methods}

\section{Strains and plasmids}

The RN4220 strain, the pCN51 expression vector, and genomic DNA from $S$. aureus strain 8325 were gifts from Dr. Gordon Archer, Virginia Commonwealth University. The pMAD shuttle vector for knockout of $k s g A$ was a gift from Dr. Gail Christie, Virginia Commonwealth University.

We constructed a $k s g A$ knockout of the $S$. aureus RN4220 strain according to the method of Arnaud et al [20]. Allelic replacement was performed using the primers in Additional file 3; chromosomal knockout was confirmed by PCR.

The $k s g A$ gene was amplified from genomic DNA from $S$. aureus strain 8325 , adding a ribosome binding sequence to ensure translation; primers used for cloning are shown in Additional file 3. The resulting fragment was subcloned into the pCN51 expression vector to produce $\mathrm{pCN}-\mathrm{WT}$. Mutagenesis was performed on this plasmid according to the Stratagene Quikchange protocol to produce $\mathrm{pCN}-\mathrm{E} 79 \mathrm{~A}$. The pCN51 constructs were transformed into strain RN4220 (RN) and the $k s g A$ knockout strain $(\triangle k s g A)$ by electroporation. Expression of active protein from the pCN51-KsgA plasmid was confirmed in the $\Delta k s g A$ strain by the kasugamycin resistance assay (Additional file 4), as well as by showing that $30 \mathrm{~S}$ subunits purified from this strain were not able to be further methylated by KsgA (Additional file 5).

\section{Antibiotic resistance assay}

Cells were grown in tryptic soy broth (TSB) at $37^{\circ} \mathrm{C}$ overnight; saturated culture was subcultured to an $\mathrm{OD}_{600}$ of 0.02 in TSB and grown with shaking at $225 \mathrm{rpm}$ to an $\mathrm{OD}_{600}$ of 0.6-0.8. The culture was then diluted 1:100 and plated onto varying concentrations of antibiotic. Plates were grown at $37^{\circ} \mathrm{C}$ overnight; the minimal inhibitory concentration (MIC) was read as the lowest concentration of antibiotic which prevented growth.

\section{Activity assay}

30 S subunits were prepared from the $S$. aureus RN4220 and $\Delta k s g A$ strains as well as from an E. coli wild-type strain. Cells were grown in TSB (S. aureus) or LB ( $E$. coli) to mid-log phase. Cells were harvested and the cell pellet resuspended in Buffer I $(50 \mathrm{mM}$ Tris, $\mathrm{pH} 7.4$, $100 \mathrm{mM} \mathrm{NH}_{4} \mathrm{Cl}, 10 \mathrm{mM} \mathrm{MgOAc}$, and $6 \mathrm{mM} \beta$-mercaptoethanol). Glass beads (0.090-0.135 mm, Thomas Scientific) were added to a final concentration of $1 \mathrm{mg} / \mu \mathrm{l}$ and the suspension was vortexed for 10 minutes. The lysates were cleared by centrifugation at $4{ }^{\circ} \mathrm{C}$, layered onto 1.1 $\mathrm{M}$ sucrose in Buffer II (50 mM Tris, $\mathrm{pH} 7.4,1 \mathrm{M}$ $\mathrm{NH}_{4} \mathrm{Cl}, 10 \mathrm{mM} \mathrm{MgOAc}$, and $6 \mathrm{mM} \beta$-mercaptoethanol), and spun in a 70Ti rotor at 35,000 rpm for 22 hours at $4^{\circ} \mathrm{C}$. The pellet of ribosomal material was resuspended in Buffer III (50 mM Tris, $\mathrm{pH}$ 7.4, $500 \mathrm{mM} \mathrm{NH}_{4} \mathrm{Cl}$, $2 \mathrm{mM} \mathrm{MgOAc}$, and $6 \mathrm{mM} \beta$-mercaptoethanol) and loaded onto a $10-40 \%$ sucrose gradient in Buffer III. The gradients were spun in an SW-28 rotor at 19,000 rpm 
for 17 hours at $4^{\circ} \mathrm{C}$ and $30 \mathrm{~S}$ fractions were collected, dialyzed into Buffer $\mathrm{K}$ (50 mM Tris, pH 7.4, $500 \mathrm{mM}$ $\mathrm{NH}_{4} \mathrm{Cl}, 2 \mathrm{mM}$ MgOAc, and $6 \mathrm{mM} \beta$-mercaptoethanol) and stored at $-80^{\circ} \mathrm{C}$. E. coli $\mathrm{KsgA}$ was purified as previously described; activity assays were performed as previously described [21].

\section{Growth experiments}

Cells were grown in TSB at $37^{\circ} \mathrm{C}$ overnight; cultures of strains transformed with $\mathrm{pCN}$ constructs included erythromycin $(10 \mu \mathrm{g} / \mathrm{ml})$. Saturated culture was subcultured to an $\mathrm{OD}_{600}$ of 0.1 in TSB; media contained cadmium $(2 \mu \mathrm{M})$ and erythromycin $(10 \mu \mathrm{g} / \mathrm{ml})$ for experiments with the $\mathrm{pCN}$ constructs. Cells were incubated with shaking (225 rpm) and the $\mathrm{OD}_{600}$ was monitored. Data were fit to an exponential growth model using the Graphpad Prism software and doubling times were calculated from the equation $\mathrm{Y}=\mathrm{Y}_{0 .} \times \mathrm{e}^{\mathrm{K} \times \mathrm{X}}$.

\section{Polysome analysis}

Cells were grown in TSB, containing cadmium $(2 \mu \mathrm{M})$ and erythromycin $(50 \mu \mathrm{g} / \mathrm{ml})$ as appropriate, to mid-log phase. Cells were harvested and the cell pellet resuspended in Buffer PA $\mu \mathrm{g} / \mathrm{ml}$ (20 mM Tris, $\mathrm{pH} 7.8,100 \mathrm{mM}$ $\mathrm{NH}_{4} \mathrm{Cl}, 10 \mathrm{mM} \mathrm{MgCl}$, and $6 \mathrm{mM} \beta$-mercaptoethanol). Glass beads (0.090-0.135 mm, Thomas Scientific) were added to a final concentration of $1 \mathrm{mg} / \mu \mathrm{l}$ and the suspension was vortexed for 10 minutes. The lysates were cleared by centrifugation at $4{ }^{\circ} \mathrm{C}$ and loaded onto a $10-40 \%$ sucrose gradient in Buffer PA. The gradients were spun in an SW-28 rotor at 19,000 rpm for 17 hours at $4^{\circ} \mathrm{C}$. Gradients were analyzed at $254 \mathrm{~nm}$ using a Biocomp Piston Gradient Fractionator with a BIORAD Econo UV Monitor with a Full Scale of 1.0. Data were recorded using DataQ DI-158-UP data acquisition software and the $70 S$ peaks were then normalized to 1 .

\section{Additional files}

Additional file 1: Growth curves of RN and $\triangle k s g A$ strains. Data represent experiments performed in triplicate; error bars indicate standard deviation.

Additional file 2: Growth curves of pCN constructs. Data represent experiments performed in triplicate; error bars indicate standard deviation.

Additional file 3: Primers used in knockout construction, KsgA cloning, and mutagenesis.

Additional file 4: Antibiotic resistance of RN4220, $\Delta \mathrm{ksgA}$, and $\triangle \mathrm{ksgA}+\mathrm{pCN} 51-\mathrm{KsgA}$ strains.

Additional file 5: Activity assay. Experiments were performed in triplicate; error bars indicate standard deviation.

\section{Competing interests}

The authors declare that they have no competing interests.

\section{Authors' contributions}

HCO carried out all experiments and drafted the manuscript. JPR conceived of the study, participated in its design and coordination, participated in construction of the knockout strain, and helped to draft the manuscript. All authors read and approved the final manuscript.

\section{Acknowledgements}

The authors would like to thank Dr. Gail Christie and Dr. Gordon Archer for providing strains and plasmids and Kristin Lane and Dr. Sam Boundy for assistance in gene knockout and expression in S. aureus.

Received: 12 July 2012 Accepted: 11 October 2012

Published: 24 October 2012

\section{References}

1. Kaczanowska M, Ryden-Aulin M: Ribosome biogenesis and the translation process in Escherichia coli. Microbiol Mol Biol Rev 2007, 71(3):477-494.

2. Helser TL, Davies JE, Dahlberg JE: Mechanism of kasugamycin resistance in Escherichia coli. Nat New Biol 1972, 235(53):6-9.

3. Connolly K, Rife JP, Culver G: Mechanistic insight into the ribosome biogenesis functions of the ancient protein KsgA. Mol Microbiol 2008, 70(5):1062-1075.

4. Ochi K, Kim JY, Tanaka Y, Wang G, Masuda K, Nanamiya H, Okamoto S, Tokuyama S, Adachi Y, Kawamura F: Inactivation of KsgA, a 16S rRNA methyltransferase, causes vigorous emergence of mutants with highlevel kasugamycin resistance. Antimicrob Agents Chemother 2009, 53(1):193-201.

5. Tufariello JM, Jacobs WR Jr, Chan J: Individual Mycobacterium tuberculosis resuscitation-promoting factor homologues are dispensable for growth in vitro and in vivo. Infect Immun 2004, 72(1):515-526.

6. Mecsas J, Bilis I, Falkow S: Identification of attenuated Yersinia pseudotuberculosis strains and characterization of an orogastric infection in BALB/c mice on day 5 postinfection by signature-tagged mutagenesis. Infect Immun 2001, 69(5):2779-2787.

7. Binet $R$, Maurelli AT: The chlamydial functional homolog of KsgA confers kasugamycin sensitivity to Chlamydia trachomatis and impacts bacterial fitness. BMC Microbiol 2009, 9:279.

8. McGhee GC, Sundin GW: Evaluation of kasugamycin for fire blight management, effect on nontarget bacteria, and assessment of kasugamycin resistance potential in Erwinia amylovora. Phytopathology 2011, 101(2):192-204.

9. Zarubica T: Specificity determinants of ArmS, a ribosomal RNA methyltransferase that confers antibiotic resistance. PhD thesis. USA: Virginia Commonwealth University, Department of Biochemistry and Molecular Biology; 2010.

10. Helser TL, Davies JE, Dahlberg JE: Change in methylation of $16 \mathrm{~S}$ ribosomal RNA associated with mutation to kasugamycin resistance in Escherichia coli. Nat New Biol 1971, 233(35):12-14.

11. Lafontaine D, Vandenhaute J, Tollervey D: The $18 \mathrm{~S}$ rRNA dimethylase Dim $1 p$ is required for pre-ribosomal RNA processing in yeast. Genes Dev 1995, 9(20):2470-2481.

12. Condon C: RNA processing and degradation in Bacillus subtilis. Microbiol Mol Biol Rev 2003, 67(2):157-174.

13. Bergman MA, Loomis WP, Mecsas J, Starnbach MN, Isberg RR: CD8(+) T cells restrict Yersinia pseudotuberculosis infection: bypass of antiphagocytosis by targeting antigen-presenting cells. PLoS Pathog 2009, 5(9):e1000573.

14. Shah DH, Zhou X, Kim HY, Call DR, Guard J: Transposon mutagenesis of Salmonella Enteritidis identifies genes that contribute to invasiveness in human and chicken cells and survival in egg albumen. Infect Immun. in press.

15. McCoy LS, Xie Y, Tor Y: Antibiotics that target protein synthesis. Wiley Interdiscip Rev RNA 2011, 2(2):209-232.

16. Comartin DJ, Brown ED: Non-ribosomal factors in ribosome subunit assembly are emerging targets for new antibacterial drugs. Curr Opin Pharmacol 2006, 6(5):453-458.

17. Campbell TL, Henderson J, Heinrichs DE, Brown ED: The yjeQ gene is required for virulence of Staphylococcus aureus. Infect Immun 2006, 74(8):4918-4921.

18. Clatworthy AE, Pierson E, Hung DT: Targeting virulence: a new paradigm for antimicrobial therapy. Nat Chem Biol 2007, 3(9):541-548. 
19. Barczak AK, Hung DT: Productive steps toward an antimicrobial targeting virulence. Curr Opin Microbiol 2009, 12(5):490-496.

20. Arnaud $M$, Chastanet $A$, Debarbouille $M$ : New vector for efficient allelic replacement in naturally nontransformable, low-GC-content, grampositive bacteria. Appl Environ Microbiol 2004, 70(11):6887-6891.

21. O'Farrell HC, Pulicherla N, Desai PM, Rife JP: Recognition of a complex substrate by the KsgA/Dim 1 family of enzymes has been conserved throughout evolution. RNA 2006, 12(5):725-733.

doi:10.1186/1471-2180-12-244

Cite this article as: O'Farrell and Rife: Staphylococcus aureus and

Escherichia coli have disparate dependences on KsgA for growth and ribosome biogenesis. BMC Microbiology 2012 12:244.

\section{Submit your next manuscript to BioMed Central and take full advantage of:}

- Convenient online submission

- Thorough peer review

- No space constraints or color figure charges

- Immediate publication on acceptance

- Inclusion in PubMed, CAS, Scopus and Google Scholar

- Research which is freely available for redistribution 